\title{
High Tibial Osteotomy
}

\author{
Dong Chul Lee, MD and Seong Joon Byun, MD \\ Department of Orthopedic Surgery, Yeungnam University College of Medicine, Daegu, Korea
}

High tibial osteotomy (HTO) is a widely performed procedure to treat medial knee arthrosis. In general, published studies on HTO report good longterm results with a correct patient selection and a precise surgical technique. The ideal candidate for an HTO is a middle aged patient (60 to 65 years of age), with isolated medial osteoarthritis, with good range of motion and without ligamentous instability. Some issues that need resolution remain; these include the choice between opening and closing wedge tibial osteotomy, the graft selection in opening wedge osteotomies, the type of fixation, the comparison with unicompartmental knee arthroplasty and whether HTO significantly affects a subsequent total joint replacement. Precise indication, preoperative planning, and operative technique selection are essential to achieve good results.

Key words: Osteoarthritis, High tibial osteotomy, Opening wedge osteotomy, Closed wedge osteotomy.

\section{Introduction}

First introduced by Jackson and Waugh ${ }^{1)}$ in 1961, high tibial osteotomy (HTO) has become popular by Conventry ${ }^{2)}$ since 1965 as a treatment modality for medial compartment osteoarthritis of the knee with varus deformity. The goals of HTO are twofold: 1) to reduce knee pain by transferring weight-bearing loads to the relatively unaffected lateral compartment in varus knees; and 2) to delay the need for a knee replacement by slowing or stopping destruction of the medial joint compartment. Although the employment of HTO has declined recently due to the improvement of knee arthroplasty, it is indisputable that appropriate patient selection, precise surgical planning, and various operative techniques can provide favorable treatment outcomes of $\mathrm{HTO}^{3-5)}$. The remaining areas of controversy regarding

Received December 30, 2011; Revised February 23, 2012;

Accepted April 15, 2012.

Correspondence to: Dong Chul Lee, MD.

Department of Orthopedic Surgery, Yeungnam University College of Medicine, 170 Hyeonchung-ro, Nam-gu, Daegu 705-717, Korea.

Tel: +82-53-620-3645, Fax: +82-53-628-4020

Email: dclee@med.yu.ac.kr

This is an Open Access article distributed under the terms of the Creative Commons Attribution Non-Commercial License (http://creativecommons.org/licenses/by-nc/3.0/) which permits unrestricted non-commercial use, distribution, and reproduction in any medium, provided the original work is properly cited.
HTO include the choice of opening vs. closing wedge HTO, graft selection in opening wedge HTO, type of fixation, comparative advantages over unicompartmental knee arthroplasty and the influence of HTO on subsequent knee arthroplasty ${ }^{6}$.

\section{Preoperative Assessments}

\section{Indications and Contraindications}

Appropriate patient selection is a key to a successful HTO. Primary or secondary medial compartment degenerative arthritis is the most common indication for HTO. The ideal candidate for HTO is an individual who is between 60 to 65 years of age with isolated medial osteoarthritis with a varus deformity and good range of motion (ROM) and without ligamentous instability ${ }^{6}$.

Poor prognostic factors include severe joint destruction ( $\geq$ Ahlback grade III), $\geq 65$ years of age, advanced patellofemoral arthritis, $<90^{\circ}$ of $\mathrm{ROM}, \geq 15^{\circ}$ of flexion contracture, joint instability and $\geq 1 \mathrm{~cm}$ lateral tibial thrust, $\geq 20^{\circ}$ of correction, and rheumatoid arthritis $^{7-9)}$.

Most authors agree that HTO is more appropriate than unicompartmental knee arthroplasty for overweight patients, but the influence of body mass index on the results of HTO remains controversial $^{8,10)}$.

\section{Preoperative Planning}

1) Patient assessment

Patient's age, career, level of activity, previous history of surgery on the knee, and expectation should be taken into consideration before deciding upon surgery. Closing wedge HTO may be more 
beneficial in reducing the risk of nonunion than opening wedge HTO for heavy-smoking patients ${ }^{11}$.

The ROM, degree of deformity, ligamentous instability, and leg length discrepancy should be assessed through physical examination. Valgus HTO can be performed for minor or moderate medial instability that can be caused by bone loss in medial compartment osteoarthritis. The status of the hip joint can have an influence on the medial osteoarthritis of the ipsilateral knee. Abduction of the hip that occurs during the stance period increases stress on the lateral compartment of the knee, which gives rise to the involvement of the stabilizers (gluteus maximus, tensor fascia latae, and biceps femoris) that results in higher forces on the lateral $\mathrm{knee}^{12)}$. Therefore, hip abductor muscle weakness or restriction or ankylosis of the hip joint should be treated prior to HTO.

\section{2) Radiographic assessment}

Multiple views should be obtained for preoperative radiographic assessment: bilateral weight-bearing anterior-posterior views in full extension, tunnel views with the knee in $30^{\circ}$ of flexion, Rosenberg views with the knee in $45^{\circ}$ of flexion, lateral views, and skyline views. The severity of medial osteoarthritis and bone loss can be evaluated from the anterior-posterior views and patellar height can be measured from the lateral views using InsallSalvati, Blackburne-Peel, or Caton-Deschamps index ${ }^{13)}$. A severe patella alta may necessitate the combined use of tibial tubercle osteotomy and closing/opening HTO. Lower limb alignment can be assessed from the full length radiographs of the lower extremity that visualizes the alignment of the hip, knee, and ankle

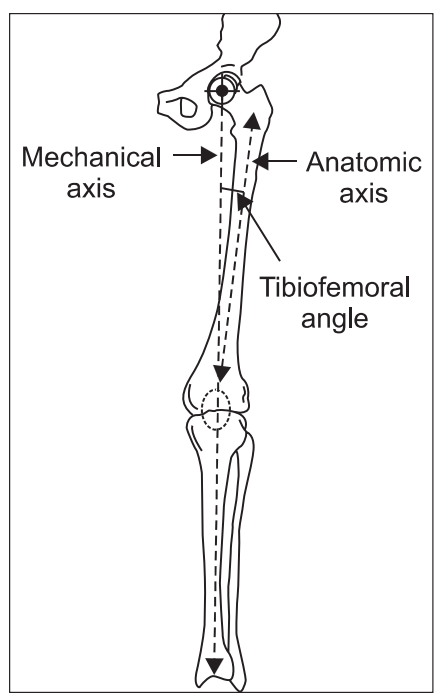

Fig. 1. A diagram of the alignment of the limb. Mechanical axis, anatomic axis and tibiofemoral angle. joints. Magnetic resonance imaging can be helpful in detecting intraosseous lesions, meniscal tears, ligamentous lesions, osteochondral defects, osteonecrosis, or subchondral edema.

\section{3) Correction angle calculation}

In normal lower extremities, the center of the hip is in line with the center of the knee and the center of the ankle and the mechanical axis, a line that connects the dots, is $0^{\circ}$ (Fig. 1). The ideal postoperative lower limb alignment is considered as $3^{\circ}-5^{\circ}$ of valgus from the mechanical axis or $8^{\circ}-10^{\circ}$ of anatomical valgus in most studies. Fujisawa et al. ${ }^{14)}$ reported that the postoperative mechanical axis should pass through the lateral one third of the tibial plateau ${ }^{4,14-16)}$. Jakob and Jacobi ${ }^{17)}$ suggested that correction of the mechanical axis depends on the thickness of the cartilage in the medial compartment: if one third of the medial cartilage is lost, the mechanical axis should pass 10-15\% lateral from the center of the tibial plateau; if two thirds of the cartilage is lost, the axis should pass $20-25 \%$ lateral; and if all is lost, the axis should pass $30-35 \%$ lateral. I often use a method described by Dugdale et al. ${ }^{18)}$ : the weight-bearing line (a line that connects the center of the femoral head and the center of the tibiotalar joint) is located at $62.5 \%$ between the medial and lateral compartment of the proximal tibia, which is slightly lateral to the lateral tibial spine
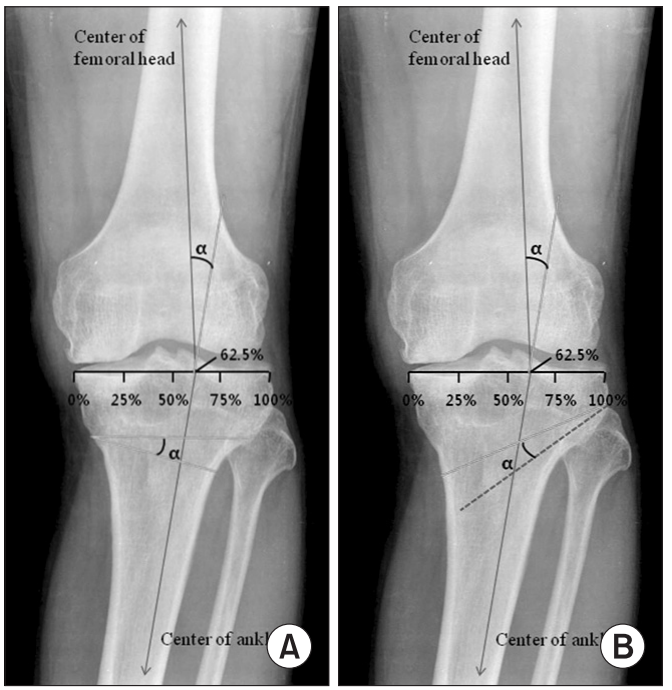

Fig. 2. Anteroposterior weight-bearing radiographs are used in planning high tibial osteotomy. (A) Closing wedge. The weight-bearing line is determined by measuring from the point located at $62.5 \%$ of the width of the tibial plateau to the center of the femoral head and the center of the ankle. The angle $(\alpha)$ formed at the intersection of these weight bearing lines represents the angle of correction. The wedge bone that constitutes the $\alpha$ angle is to be removed. (B) Opening wedge. The $\alpha$ angle is calculated and transferred to the osteotomy site to open the proximal tibia. 
and $3^{\circ}-5^{\circ}$ valgus from the mechanical axis. Correction should be planned and carried out with care because undercorrection may result in the recurrence of varus deformity and overcorrection may cause poor cosmetic and functional outcomes.

In closing wedge HTO, the correction angle $(\alpha)$ is defined as an angle formed by a line drawn from the $62.5 \%$ point of the width of the tibial plateau to the center of the femoral head and another line to the center of the ankle joint. The proximal osteotomy line is located in parallel with the articular surface and $2-2.5 \mathrm{~cm}$ inferior to the joint line The distal osteotomy line is determined referring to the $\alpha$ angle and the wedge bone between the osteotomy lines is removed (Fig. 2A).

Opening wedge HTO is planned in a similar fashion with the closing wedge HTO. The proximal osteotomy line is drawn from a point 3.5-4 cm inferior to the medial knee joint line to the tip of the fibular head from which another same length line is drawn obliquely by the $\alpha$ angle. The line that runs between the endpoints of each line is used for exposure during surgery (Fig. 2B).

In HTO for medial compartment osteoarthritis without knee instability, efforts should be made to maintain the preoperative anatomical posterior tibial slope.

Some recent clinical studies have shown that the use of navigation systems contributed to the preciseness, accuracy, and reproducibility of $\mathrm{HTO}^{19)}$.

\section{HTO Techniques}

There are various HTO techniques including closing wedge osteotomy, opening wedge osteotomy, dome osteotomy, progressive callus distraction, and chevron osteotomy. Of these, opening wedge HTO and closing wedge HTO are most commonly performed ${ }^{6,20)}$.

\section{Medial Opening Wedge Osteotomy \\ 1) Advantages and disadvantages}

Medial opening wedge osteotomy is a relatively simple procedure that involves a single osteotomy and a few dissections.

The technique does not necessitate either a fibular osteotomy that has been associated with neurovascular complications or bone resection of the lateral tibia. Accordingly, the normal anatomical tibial bone shape is maintained after the procedure, which allows for conversion to knee replacement. The level of correction can be identified and adjusted intraoperatively on the coronal and sagittal planes and shortening of the lower limbs can be prevented or treated.

However, the technique has been associated with high nonunion rates, long period of weight-bearing restriction, and leg lengthening. Other disadvantages include harvest site morbidity, loss of correction due to unstable fixation, and increase in posterior tibial slope due to anterior position of the metal plate.

\section{2) Good candidates for medial opening wedge osteotomy}

Medial opening wedge osteotomy can be more effective than closing wedge HTO in knees with $\geq 2 \mathrm{~mm}$ leg length discrepancy, a combined posterior cruciate ligament injury, patella alta, or medial collateral ligament laxity ${ }^{21)}$.

\section{3) Surgical technique}

The patient is placed in the supine position on a radiolucent operating table and a tourniquet is applied. An arthroscopy can
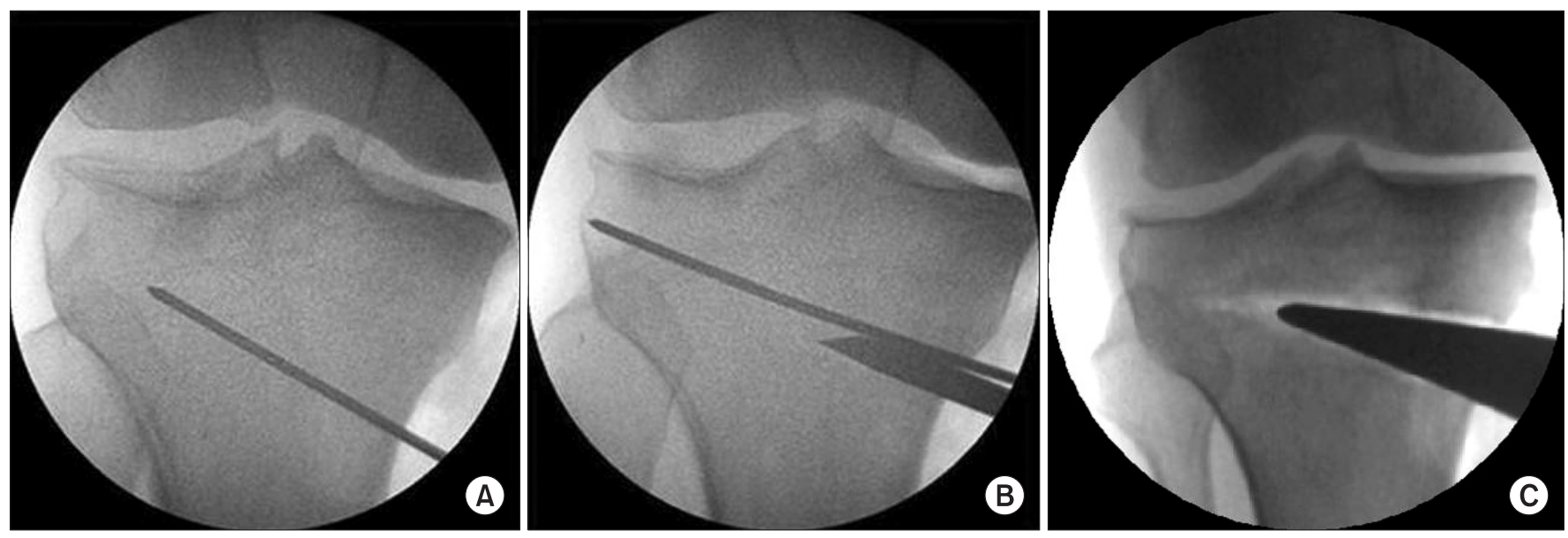

Fig. 3. Opening wedge osteotomy. (A) A guide wire is placed from $3.5-4 \mathrm{~cm}$ below the medial joint line to about $1 \mathrm{~cm}$ below the lateral articular margin of the tibia. (B) Cortical osteotomy is performed with an oscillating saw inferior to the guide wire and an osteotome. (C) When the osteotomy is completed, the medial tibia is opened with a wedge. 

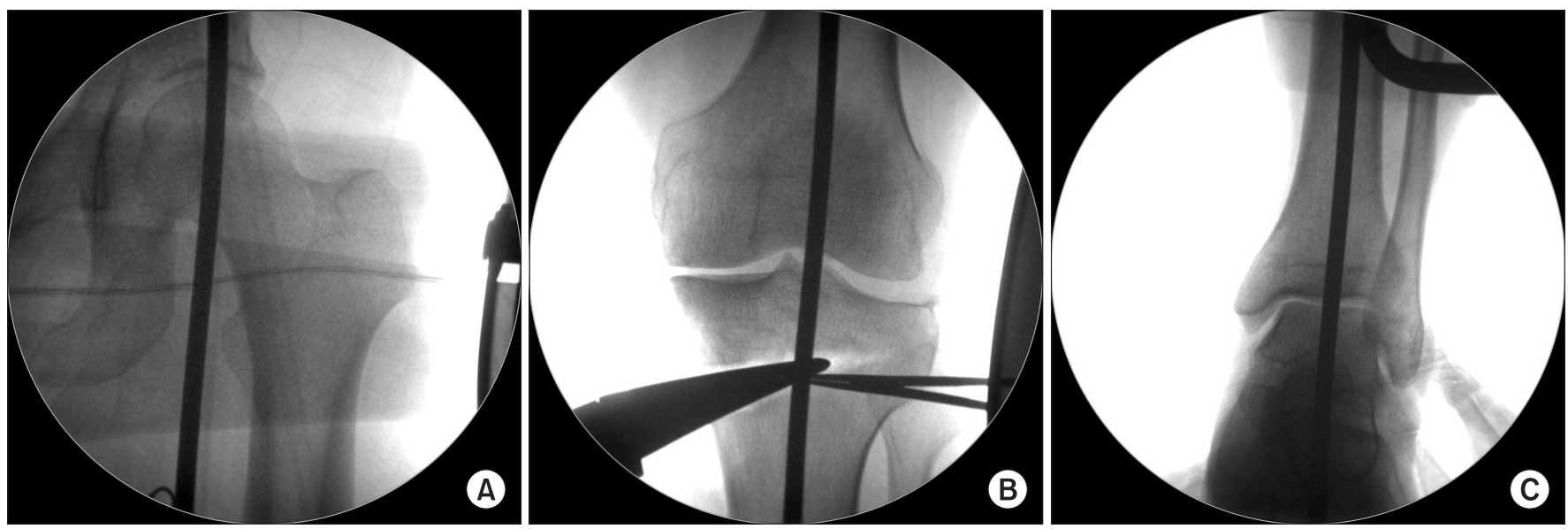

Fig. 4. An image intensifier and an alignment rod are used to control proper coronal alignment during axial loading of the joint. (A) The center of the femoral head. (B) The point (located at $62.5 \%$ of the width of the tibial plateau) on the tibial plateau. (C) The center of the ankle.

be used at the same time to diagnose and treat intraarticular lesions. A 5-cm vertical incision is made over the center between the medial aspect of the tibial tuberosity and the posteromedial aspect of the tibia below the joint line. The pes anserinus is detached from the tibia to expose the superficial medial collateral ligament. The distal portion of the exposed ligament is separated from bone and a blunt retractor is inserted posterior to the medial collateral ligament and the tibia to protect the neurovascular structures posterior to the incision line. After identifying the medial border of the patellar tendon, subperiosteal dissection is performed from the tibial tuberosity to the posteromedial aspect of the tibia. Two guide wires are inserted at a point $3.5-4 \mathrm{~cm}$ below the medial joint line and passed obliquely $1 \mathrm{~cm}$ below the lateral articular margin of the tibia towards the tip of the fibular head (Fig. 3A). After checking the appropriate location with fluoroscope, a tibial osteotomy is performed immediately below the guide wires using an oscillating saw or an osteotome (Fig. 3B). Ensure the osteotomy line extends from the tibial tuberosity along the posteromedial aspect of the tibia to $1 \mathrm{~cm}$ medial to the the lateral tibial cortex and is in parallel with the posterior tibial slope on the sagittal plane ${ }^{20)}$. The mobility of the osteotomy site is checked and the osteotomy is opened with a valgus force. If the opening of the osteotomy seems insufficient, use 2 or 3 stacked osteotomes to reduce the risk of intraarticular fractures ${ }^{20)}$. Subsequently, a calibrated wedge is inserted until the osteotomy is opened to the desired extent (Fig. 3C).

Ensure with fluoroscopy when a long alignment rod or wire cable is centered over the hip joint (Fig. 4A) and the ankle joint (Fig. 4C), it lies at $62.5 \%$ of the width of the tibial plateau (Fig. 4B).

Once the desired degree of correction is achieved, internal fixation of a metal plate is performed. There are various types of metal plates including the Puddu plate, Tomofix, Aesculap (dual) plate, -plates with or without a spacer (rectangular or tapered $)^{10)}$. Among these, spacer plates are most commonly used and the metal block should be identical to the calibrated wedge. The proximal fixation screws should be used under fluoroscopic guidance and the defect should be grafted using iliac crest autograft, allograft, or a bone substitute ${ }^{10)}$. For defects $\geq 10 \mathrm{~mm}$, corticocancellous autografts or allografts are used, whereas for small defects, bone grafting is optional.

\section{Lateral Closing Wedge Osteotomy}

\section{1) Advantages and disadvantages}

Lateral closing wedge osteotomy is effective for correction near maximal point of deformity. The technique allows rapid bone union due to the large contact surface of cancellous bone at the osteotomy site, early weight bearing and rehabilitation, and the use of quadriceps femoris muscle force. In addition, the articular surface can be observed through the skin incision and the risk of loss of correction is low.

However, the procedure requires a fibular osteotomy or a release of the proximal tibiofibular joint, which can result in neurovascular complications. Lateral bone resection can cause shortening of the lower limb. In addition, stem impingement or metal augmentation is unavoidable in a subsequent total knee replacement due to the proximal tibial deformity and bone loss of the lateral condyle.

2) Good candidates for lateral closing wedge osteotomy Lateral closing wedge osteotomy is more appropriate for patients with heavy smoking, diabetes, or prolonged use of steroids that 
have been associated with the risk of nonunion. Besides, the procedure can be performed when patellar infera is present, the patient does not want bone grafting, or anterior cruciate ligament reconstruction should be carried out additionally ${ }^{22}$.

\section{3) Surgical technique}

There are various modified versions of lateral closing wedge osteotomy, but all of them are based on the same principles ${ }^{18,23,24)}$. The skin incision can be transverse, longitudinal, or L-shaped. A longitudinal incision allows for easy conversion to knee replacement, whereas a transverse incision reduces the risk of peroneal nerve palsy because tibial osteotomy and partial resection of the inferomedial fibular head can be performed through the same incision line. Although the osteotomy can be performed below the tibial tubercle, proximal tibial tubercle osteotomy offers more advantages: the metaphyseal osteotomy promotes early bone union; correction can be performed adjacent to the deformity; and quadriceps femoris muscle force on the osteotomy site improves stability of the knee. A $10-\mathrm{cm}$ vertical incision is made and the tibialis anterior muscle of the proximal tibia is elevated to expose the proximal tibiofibular joint. For the management of the proximal tibiofibular joint, joint disruption, fibular osteotomy at a site $15 \mathrm{~cm}$ distal to the fibular head, and excision of the fibular head are available.

A retractor is placed under the lateral margin of the patellar tendon for protection and another retractor is positioned along the posterolateral aspect of the tibia to protect the neurovascular structures. The osteotomy is commenced $2 \mathrm{~cm}$ below the lateral articular surface of the tibia. The proximal osteotomy should be cut in parallel with the articular surface and the distal osteotomy should be cut obliquely to prevent patellar tendon. The lateral bone wedge can be removed using an angular cutting guide. To reduce the risk of intraarticular fractures, lateral cortex and wedge should be removed by properly using a saw, roengeur, curette, or an osteotome and preserving 3-6 mm of the opposite cortical hinge. The medial tibial cortex should not be completely osteotomized but perforated by multiple holes to work as a hinge when the osteotomy is closed. The hinge should be closed slowly to produce plastic deformation. Ensure to avoid overcorrection that can occur when the proximal cortex of the distal fragment is overlapped by distal cortex of the proximal fragment.

Confirm complete wedge removal using fluoroscopy. Once the osteotomy is closed using valgus force, the alignment should be checked. An ideal correction, overcorrection of the mechanical axis by $3^{\circ}-5^{\circ}$, can be considered achieved if the rod lies at $62.5 \%$ of the width of the tibial plateau. Fixation can be performed using 1 or 2 staples from lateral to medial direction anterior to the fibula. For more rigid fixation, a contoured T-plate or locking plate can be applied ${ }^{20}$.

\section{Other Techniques}

Other HTO techniques include dome osteotomy, progressive callus distraction using an external fixator, and chevron osteotomy.

Dome osteotomy or progressive callus distraction is indicated when a large degree of correction involving $18-20 \mathrm{~mm}$ opening or closing or $\geq 20^{\circ}$ angular correction is necessary for traumatic varus deformity or Blount disease. Dome osteotomy is a procedure using an inverse U-shaped proximal tibial bone cut and a metal plate for fixation or an external fixator for progressive correction. This technique is useful for achieving correction without any changes in the patellar height.

The progressive callus distraction is performed through an opening wedge osteotomy and external fixation using an axial or ring fixator. Most progressive callus distraction techniques are used for large degree of correction and require an osteotomy distal to tibial tubercle to minimize changes in the patellar height. The use of an external fixator allows for correction of various axes, early rehabilitation and weight bearing, and microalignment of the lower limbs. However, external fixation can cause discomfort, pin infections, loss of correction after fixator removal.

Chevron osteotomy is a procedure in which an inverse V-shaped bone cut is made, a lateral wedge is inserted medially, and rigid metal plate fixation is performed. Although this technique does not cause bone loss or require bone grafting, it has not been frequently employed due to its technical difficulty and invasiveness.

\section{Other Indications}

\section{Varus Malalignment in Meniscectomized Knees}

Osteoarthritis may develop in meniscectomized knees due to long-term overload. Restoration of the normal lower limb alignment would reduce the load on the joint and the damage to the cartilage and graft. Therefore, HTO and meniscal transplantation can be performed at the same time in young patients with meniscectomy.

\section{Varus Malalignment and Chondral Resurfacing}

Lower limb malalignment may result in poor outcomes after a cartilage repair including microfracture, mosaicplasty, 
osteochondral allograft transplantation, or autologous chondrocyte implantation. The concomitant use of chondral resurfacing and HTO has not been proven as an effective method for improving clinical results. However, I believe correction of the varus alignment during chondral resurfacing would contribute to achieving good treatment outcomes.

\section{Rehabilitation}

Once adequate fixation is achieved after medial opening wedge osteotomy, early joint exercises should be initiated. Partial weight bearing exercises should be performed with the knee protected in a hinged brace allowing $0^{\circ}-90^{\circ}$ of motion for 6 weeks $s^{6}$. Weight bearing should be increased progressively between 6 to 12 weeks with the knee brace removed. Maintenance of correction and bone union should be assessed through radiography on a regular basis and the lower limb alignment on full length radiographs of the lower extremity at 6 months postoperatively ${ }^{20)}$. After lateral closing wedge osteotomy, partial weight bearing exercises are allowed immediately after surgery. A hinged knee brace should be worn for 6 postoperative weeks and weight bearing can be increased progressively depending on the state of bone union.

\section{Posterior Tibial Slope and Patellar Height after HTO}

HTO can result in changes in patellar height and posterior tibial slope.

Lateral closing wedge osteotomy causes an elevation of the tibial tuberocity due to shortening of the proximal tibia during the procedure, which increases patellar height and is useful for patella baja (Fig. 5A, 5B ${ }^{25,26}$. Medial opening wedge osteotomy causes a
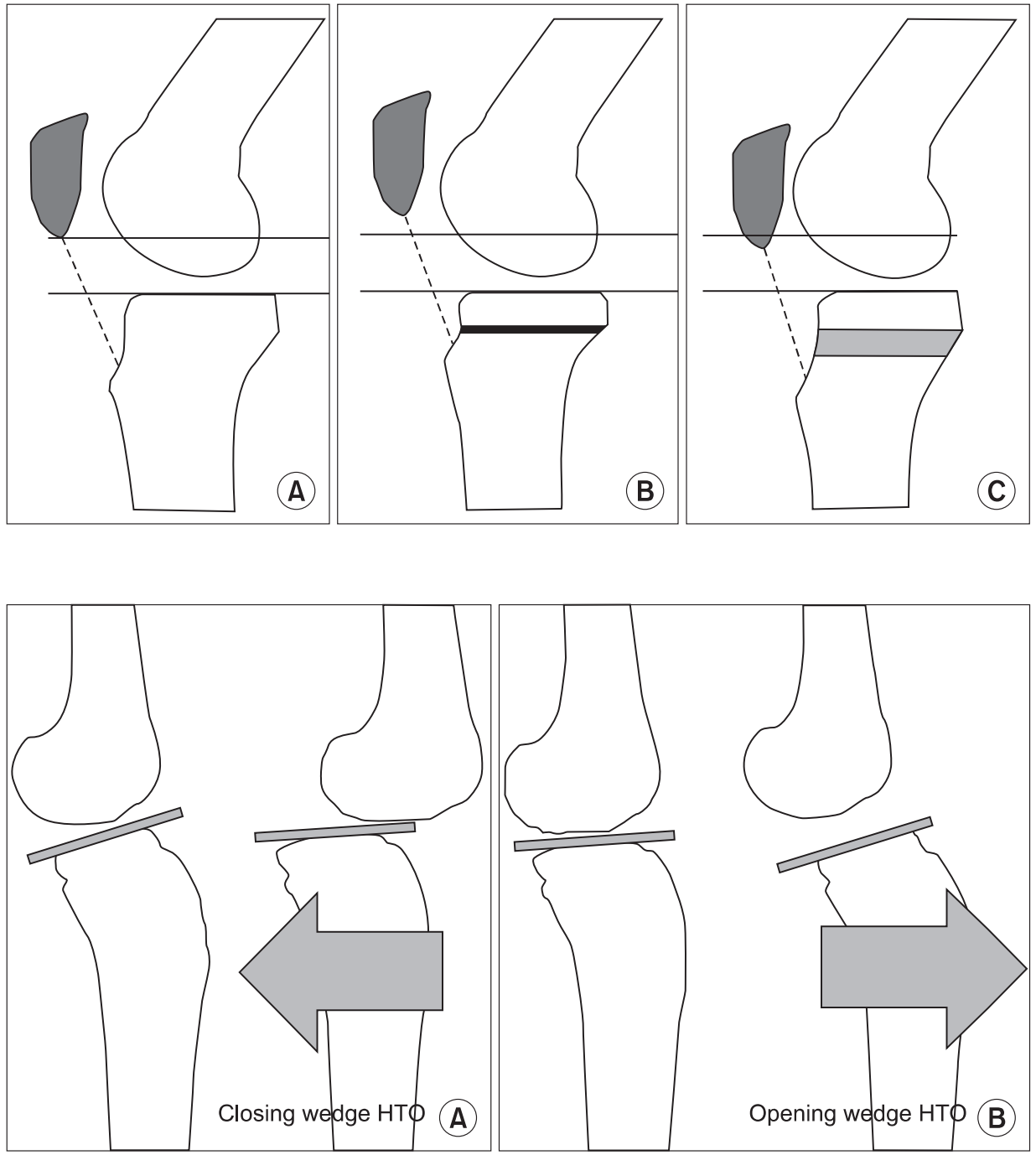

Fig. 5. The level of the patella after closing and opening wedge high tibial osteotomy. (A) Preoperatively. (B) After closing wedge osteotomy, the segment between the tibial plateau and tibial tuberosity is shortened. (C) After opening wedge osteotomy, this segment is enlongated. Relative patella baja can occur.
Fig. 6. Change of the tibial slope after high tibial osteotomy. (A) Closing wedge high tibial osteotomy (HTO) can cause no change or a decrease in posterior tibial slope, and posterior translation of the tibia can be increased. (B) Opening wedge HTO usually can be caused an increase in posterior tibial slope, and anterior translation of the tibia can be increased. 
decrease in patellar height because the tibial tuberosity is lowered due to opening of the proximal tibia during the procedure (Fig. $5 \mathrm{C})^{27)}$.

Lateral closing wedge osteotomy can result in a decrease in posterior tibial slope that causes hyperextension and overload on the posterior cruciate ligament (PCL), which contributes to reduction in anterior instability (Fig. 6A). Medial opening wedge osteotomy heightens the likelihood of increased posterior slope that restricts extension and causes overload on the ACL. Accordingly, this procedure is recommended in knees with chronic PCL injuries and posterolateral instability that has been inversely correlated with posterior tibial slope (Fig. 6B). Care should be taken not to cause the posterior tibial slope to be $\geq 10^{\circ}$ because the load on the ACL is increased by more than 3 times in this case ${ }^{28}$. Song et al. ${ }^{29)}$ suggested that the anterior opening gap should be $67 \%$ of the posterior gap in order to maintain the posterior tibia slope.

\section{Clinical Outcomes}

There is a consensus among authors that slight overcorrection in HTO produces more satisfying results. However, the optimal degree of valgus angulation is still controversial. Dugdale et al. ${ }^{18)}$ recommended $3^{\circ}-5^{\circ}$ valgus mechanical axis, whereas other authors suggested $3^{\circ}-6^{030)}, 3^{\circ}-7^{010)}$, or $7^{\circ}-9^{023)}$. Conventry et al..$^{31)}$ reported that the 10 -year survival rates were $63 \%$ in knees with $5^{\circ}$ valgus angulation, $87 \%$ in knees with $6^{\circ}-7^{\circ}$ valgus angulation, and $94 \%$ in knees with $\geq 8^{\circ}$ valgus angulation.

Brouwer et al. ${ }^{32)}$ reported that lateral closing wedge osteotomy improved knee function and reduced pain for medial compartment arthritis of the knee. In addition, there were no significant differences between the opening and closing wedge techniques in terms of pain, function, and complications during the 1 year follow-up.

Some authors raise concern that conversion to total knee arthroplasty (TKA) after HTO can cause adhesion or shortening of the patellar tendon or bone loss or overcorrection due to tibial plateau deformity. However, many long-term follow-up studies have failed to demonstrate significant clinical differences between the knees with a primary TKA and a TKA following an $\mathrm{HTO}^{10}$. In the study by van Raaij et al. ${ }^{33)}$, TKA after lateral closing wedge osteotomy required prolonged operation time and extra operative procedures and caused some reduction in postoperative knee ROM. On the other hand, the osteotomy did not have significant influences on clinical outcomes in the mid- and long-term follow-up. Theoretically, opening wedge osteotomy is advantageous over closing wedge osteotomy regarding bone stock loss and impingement between the tibial component stem and the anterior tibial cortex ${ }^{10)}$.

\section{Complications}

Fracture of the medial or lateral tibial cortical hinge and intraarticular fracture are commonly encountered after HTO, which can disrupt stability and healing at the osteotomy site and congruence of the articular surface. In these cases, the use of locking metal plates or extra screws and metal plates can provide stale fixation for reduction of the risk of loss of correction and nonunion ${ }^{10)}$. The incidence of nonunion after HTO has been reported to be $0.7-4.4 \%{ }^{34)}$. The risk factors for nonunion include large degree of correction in HTO, smoking, and insufficient fixation.

Due to the recent advancement in internal fixation techniques, early joint exercises are allowed after HTO. In the past, longterm cast immobilization was unavoidable after closing HTO, during immobilization patellar infera secondary to patellar tendon contracture could occur with a prevalence of $7.6-8.8 \%{ }^{27}$. In addition, severe patella infera has a negative influence on the prognosis of HTO and renders conversion to TKA difficult.

The incidence of common peroneal nerve palsy caused by nerve damage during HTO is 2-16\% and fibular shaft osteotomy (at $15 \mathrm{~cm}$ distal to the fibular head) can be useful for reduction of such damage ${ }^{34)}$. The reported rate of infection following external fixation is $2.3-54.5 \%$, whereas that of infection following internal fixation is $\leq 4 \%$. Other possible complications include fixation failure, loss of correction, pseudoarthrosis, deep venous thrombosis, pulmonary embolism, and compartment syndrome ${ }^{34)}$.

\section{Conclusions}

HTO is a procedure performed to treat medial knee arthrosis in young or middle-aged active patients. Appropriate patient selection, proper osteotomy types, and precise surgical techniques are essential to success of HTO. Drawbacks of the procedure are restricted motion during the bone union period and possibility of delayed union or nonunion. On the other hand, participation in aggressive activities is allowed after bone union and the reported short-term follow-up clinical results are excellent in $80-90 \%$ of the patients. In addition, the successful outcome of HTO can be maintained for more than 8 to 10 years delaying the need for conversion to TKA. Therefore, I think HTO should be 
recommended for the treatment of degenerative arthritis of the knee in young, active patients for symptomatic improvement and maintenance of activity levels.

\section{References}

1. Jackson JP, Waugh W. Tibial osteotomy for osteoarthritis of the knee. J Bone Joint Surg Br. 1961;43:746-51.

2. Conventry MB. Osteotomy of the upper portion of the tibia for degenerative arthritis of the knee. A preliminary report. J Bone Joint Surg Am. 1965;47:984-90.

3. Yasuda K, Majima T, Tsuchida T, Kaneda K. A ten- to 15year follow-up observation of high tibial osteotomy in medial compartment osteoarthrosis. Clin Orthop Relat Res. 1992;(282):186-95.

4. Bauer GC, Insall J, Koshino T. Tibial osteotomy in gonoarthrosis (osteo-arthritis of the knee). J Bone Joint Surg Am. 1969;51:1545-63.

5. Insall JN, Joseph DM, Msika C. High tibial osteotomy for varus gonathrosis. A long-term follow-up study. J Bone Joint Surg Am. 1984;66:1040-8.

6. Rossi R, Bonasia DE, Amendola A. The role of high tibial osteotomy in the varus knee. J Am Acad Orthop Surg. 2011;19:590-9.

7. Aglietti P, Rinonapoli E, Stringa G, Taviani A. Tibial osteotomy for the varus osteoarthritic knee. Clin Orthop Relat Res. 1983:(176):239-51.

8. Naudie D, Bourne RB, Rorabeck CH, Bourne TJ. The Install Award. Survivorship of the high tibial valgus osteotomy. A 10- to 22-year followup study. Clin Orthop Relat Res. 1999;(367):18-27.

9. Rudan JF, Simurda MA. High tibial osteotomy. A prospective clinical and roentgenographic review. Clin Orthop Relat Res. 1990;(255):251-6.

10. Amendola A, Bonasia DE. Results of high tibial osteotomy: review of the literature. Int Orthop. 2010;34:155-60.

11. A WD, Toksvig-Larsen S. Cigarette smoking delays bone healing: a prospective study of 200 patients operated on by the hemicallotasis technique. Acta Orthop Scand. 2004;75:347-51.

12. Hinman RS, Hunt MA, Creaby MW, Wrigley TV, McManus FJ, Bennell KL. Hip muscle weakness in individuals with medial knee osteoarthritis. Arthritis Care Res (Hoboken). 2010;62:1190-3.

13. Phillips CL, Silver DA, Schranz PJ, Mandalia V. The measurement of patellar height: a review of the methods of imaging.
J Bone Joint Surg Br. 2010;92:1045-53.

14. Fujisawa $\mathrm{Y}$, Masuhara K, Shiomi S. The effect of high tibial osteotomy on osteoarthritis of the knee. An arthroscopic study of 54 knee joints. Orthop Clin North Am. 1979;10:585608.

15. MacIntosh DL, Welsh RP. Joint debridement-a complement to high tibial osteotomy in the treatment of degenerative arthritis of the knee. J Bone Joint Surg Am. 1977;59:1094-7.

16. Conventry MB. Upper tibial osteotomy for osteoarthritis. J Bone Joint Surg Am. 1985;67:1136-40.

17. Jakob RP, Jacobi M. Closing wedge osteotomy of the tibial head in treatment of single compartment arthrosis. Orthopade. 2004;33:143-52.

18. Dugdale TW, Noyes FR, Styer D. Preoperative planning for high tibial osteotomy. The effect of lateral tibiofemoral separation and tibiofemoral length. Clin Orthop Relat Res. 1992;(274):248-64.

19. Saragaglia D, Roberts J. Navigated osteotomies around the knee in 170 patients with osteoarthritis secondary to genu varum. Orthopedics. 2005;28:s1269-74.

20. Amendola A. Unicompartmental osteoarthritis in the active patient: the role of high tibial osteotomy. Arthroscopy. 2003; 19 Suppl 1:109-16.

21. Giffin JR, Stabile KJ, Zantop T, Vogrin TM, Woo SL, Harner CD. Importance of tibial slope for stability of the posterior cruciate ligament deficient knee. Am J Sports Med. 2007;35:1443-9.

22. Savarese E, Bisicchia S, Romeo R, Amendola A. Role of high tibial osteotomy in chronic injuries of posterior cruciate ligament and posterolateral corner. J Orthop Traumatol. 2011;12:1-17.

23. Conventry MB, Bowman PW. Long-term results of upper tibial osteotomy for degenerative arthritis of the knee. Acta Orthop Belg. 1982;48:139-56.

24. Insall J, Shoji H, Mayer V. High tibial osteotomy. A five-year evaluation. J Bone Joint Surg Am. 1974;56:1397-405.

25. Lerat JL, Moyen B, Garin C, Mandrino A, Besse JL, BrunetGuedj E. Anterior laxity and internal arthritis of the knee. Results of the reconstruction of the anterior cruciate ligament associated with tibial osteotomy. Rev Chir Orthop Reparatrice Appar Mot. 1993;79:365-74.

26. Hohmann E, Bryant A, Imhoff AB. The effect of closed wedge high tibial osteotomy on tibial slope: a radiographic study. Knee Surg Sports Traumatol Arthrosc. 2006;14:454-9.

27. Wright JM, Crockett HC, Slawski DP, Madsen MW, Windsor RE. High tibial osteotomy. J Am Acad Orthop 
Surg. 2005;13:279-89.

28. Dejour H, Bonnin M. Tibial translation after anterior cruciate ligament rupture. Two radiological tests compared. J Bone Joint Surg Br. 1994;76:745-9.

29. Song EK, Seon JK, Park SJ, Jeong MS. The complications of high tibial osteotomy: closing- versus opening-wedge methods. J Bone Joint Surg Br. 2010;92:1245-52.

30. Hernigou P, Medevielle D, Debeyre J, Goutallier D. Proximal tibial osteotomy for osteoarthritis with varus deformity. A ten to thirteen-year follow-up study. J Bone Joint Surg Am. 1987;69:332-54.

31. Conventry MB, Ilstrup DM, Wallrichs SL. Proximal tibial osteotomy. A critical long-term study of eighty-seven cases. J
Bone Joint Surg Am. 1993;75:196-201.

32. Brouwer RW, Bierma-Zeinstra SM, van Raaij TM, Verhaar JA. Osteotomy for medial compartment arthritis of the knee using a closing wedge or an opening wedge controlled by a Puddu plate. A one-year randomised, controlled study. J Bone Joint Surg Br. 2006;88:1454-9.

33. van Raaij TM, Reijman M, Furlan AD, Verhaar JA. Total knee arthroplasty after high tibial osteotomy. A systematic review. BMC Musculoskelet Disord. 2009;10:88.

34. Spahn G. Complications in high tibial (medial opening wedge) osteotomy. Arch Orthop Trauma Surg. 2004;124:64953. 